\title{
Convergent Mechanisms Mediate Preparatory States and Repetition Priming in the Feeding Network of Aplysia
}

\author{
Alex Proekt and Klaudiusz R. Weiss \\ Department of Physiology and Biophysics, Mount Sinai School of Medicine, New York, New York 10029-6574
}

\begin{abstract}
Improvement of behavioral responses to expected stimuli has been attributed to a change in the preparatory state. In this study, we take advantage of the accessibility of the nervous system of Aplysia and develop an in vitro analog of the preparatory state for feeding behaviors. We provide evidence that the change in the preparatory state may be elicited initially by activity of identified serotonergic metacerebral cells (MCCs). We demonstrate, however, that the preparatory state is maintained through MCC-independent repetition priming that is embedded in the properties of the behavior-generating network. Both MCC-dependent and MCC-independent processes converge on the same site in the behavior-generating network to mediate the change in the preparatory state. Thus, we propose a model of how multiple neuronal structures interact to elicit and maintain changes in the preparatory states in the CNS.
\end{abstract}

Key words: Aplysia; repetition priming; preparatory states; metacerebral cells; central pattern generator; post-tetanic potentiation; anticipation; serotonin

\section{Introduction}

Anticipatory or preparatory states are a salient feature of behavior. Changes in these internal states are used to explain the shortening of the latency of responses when stimuli are cued or otherwise expected. Hypothetically, any previous event that reliably precedes the occurrence of a stimulus can serve as a cue (for example, repetition of the same stimulus). In fact, repeated presentation of the same stimulus is typically accompanied by shortening of the latency of responses, a phenomenon referred to as repetition priming (Schacter and Buckner, 1998; Wiggs and Martin, 1998). Alternatively, cues that do not themselves trigger behaviors, but precede the onset of the stimulus reliably, can shorten the latency to subsequent stimulus-elicited responses (Connolly et al., 2002). Although these two situations are conceptually similar, it is unknown whether neurons that mediate the shortening of the latency of responses to cued stimuli and those that shorten the latency of responses to repeated stimuli engage the same mechanisms in the CNS.

Although preparatory states are commonly studied for conditioned responses (e.g., bar press), they are also prevalent in unconditioned behaviors (e.g., feeding). When animals encounter salient stimuli (e.g., food) in the environment, they often begin to orient to such stimuli. It is believed that during this orienting phase, also referred to as the appetitive phase, the preparatory state for subsequent consummatory behaviors is established. Feeding behavior of Aplysia is one behavior that consists of the appetitive phase, which is followed by the consummatory phase.

\footnotetext{
Received Jan. 16, 2003; revised March 10, 2003; accepted March 10, 2003.

This work was supported by National Institute of Mental Health Grants MH50235 and MH35564. We thank Drs. Vladimir Brezina and Elizabeth Cropper for stimulating discussion and comments on this manuscript.

Correspondence should be addressed to Dr. Klaudiusz R. Weiss, Department of Physiology and Biophysics, Box 1218, Mount Sinai School of Medicine, 1 Gustave Levy Place, New York, NY 10029. E-mail: klaudiusz.weiss@mssm.edu.

Copyright $\odot 2003$ Society for Neuroscience $\quad$ 0270-6474/03/234029-05\$15.00/0
}

The appetitive phase begins when Aplysia sense the presence of food; this phase serves to bring the animal in contact with the food. The appetitive phase is followed by the consummatory phase, which consists of repetitive biting and swallowing (Kupfermann, 1974a,b). The consummatory behaviors of Aplysia are influenced by the preparatory state of the animal, because previous exposure to food shortens the latency to subsequent biting responses (Kupfermann, 1974a; Susswein et al., 1984). It has been suggested that the serotonergic metacerebral cells (MCCs) that are active during the appetitive phase contribute to the establishment of the preparatory state for consummatory behaviors (Kupfermann et al., 1979; Kupfermann and Weiss, 1982). In addition to the MCC-elicited preparatory state, it has been suggested that the consummatory behaviors in Aplysia are influenced by MCCindependent repetition priming, referred to as food-induced arousal (Rosen et al., 1989). In this study, we first establish an in vitro analog of the preparatory state for consummatory responses. We then investigate whether the MCCs and processes responsible for MCC-independent repetition priming engage the same mechanisms in the network that mediates feeding in Aplysia.

\section{Materials and Methods}

Specimens of Aplysia californica were obtained from Marinus (Long Beach, CA). Animals weighing 100-200 gm were anesthetized by injection of isotonic $\mathrm{MgCl}_{2}$. Buccal and cerebral ganglia were dissected out, desheathed, and pinned out. The preparation was continuously perfused with artificial seawater (in mM: $460 \mathrm{NaCl}, 10 \mathrm{KCl}, 55 \mathrm{MgCl}_{2}, 11 \mathrm{CaCl}_{2}$, and 10 HEPES buffer, $\mathrm{pH}$ 7.6). All experiments were performed at $14-$ $15^{\circ} \mathrm{C}$. Standard intracellular and extracellular recording techniques were used.

PSPs were analyzed using the variance method (Hubbard et al., 1969). Amplitude of the PSPs is small relative to the distance from the their reversal potential; therefore, the amplitude of the PSPs was not corrected for nonlinearity. The method assumes that the number of quanta of 

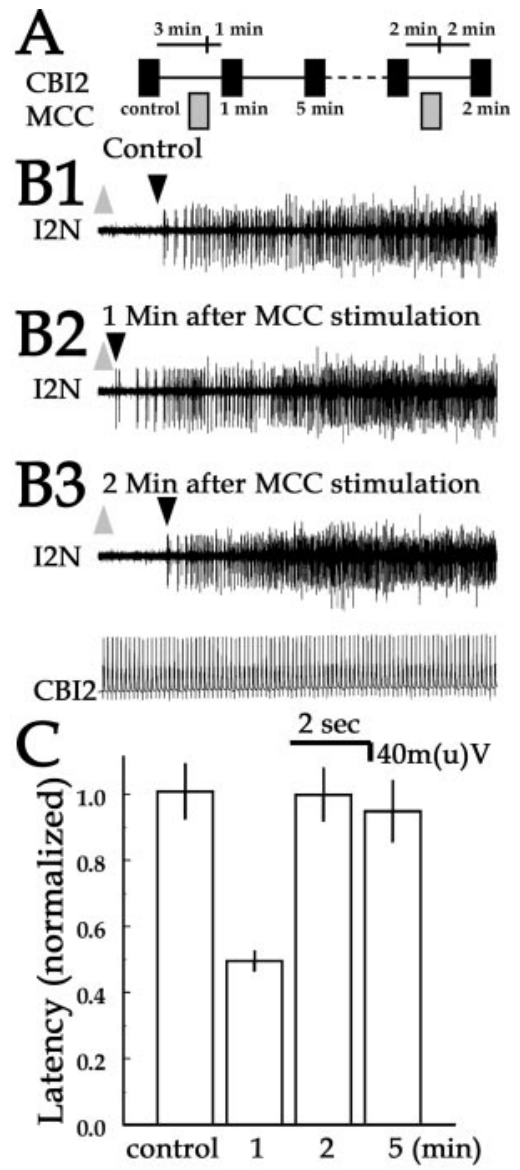

Figure 1. Effects of MCC stimulation on the latency of (BI-2-elicited CMPs. A, Paradigm used in the experiment shown in $B$. CBI-2 was stimulated at $9 \mathrm{~Hz}$ for the duration of the protraction phase to elicit one CMP every 4 min. MCC was stimulated at $8 \mathrm{~Hz}$ for $1 \mathrm{~min}$. MCC stimulation preceded $\mathrm{CBI}-2$ stimulation by either 1 or $2 \mathrm{~min}$. B, All traces are aligned with respect to the beginning of $\mathrm{CBI}-2$ stimulation, one example of which is shown at the bottom. Gray arrows indicate the onset of $\mathrm{CBI}-2$ stimulation. Black arrows indicate the onset of activity in the $\mathrm{I} 2$ nerve (I2N). B1, I2N recording from a control CMP. B2, I2N recording from a CMP elicited 1 min after MCC stimulation. B3, I2N recording from a CMP elicited 2 min after MCC stimulation. C, Grouped data from five preparations. Latency was normalized to the average of the latency to the onset of B61/62 firing of two CMPs preceding the stimulation of the MCC. Data are presented as average \pm SEM.

transmitter released from the presynaptic neuron follows a Poisson distribution. The average amplitude of miniature PSPs $(\bar{q})$ is estimated as the ratio of the variance of the amplitude of the PSP $(\operatorname{var}(v))$ to the mean of the amplitude of the PSP $(\bar{v}) \bar{q}=\operatorname{var}(v) / \bar{v}$. The number of quanta released $(m)$ are calculated by dividing $\bar{v}$ by $\bar{q}$. Change in $m$ is considered to be a presynaptic effect. The change in $\bar{q}$ is considered to be a postsynaptic effect.

\section{Results}

Consummatory behaviors in Aplysia are organized by a central pattern generator (CPG) located in the buccal ganglion. In the isolated nervous system, the buccal CPG can be activated to generate consummatory-like motor programs (CMPs) by stimulation of the command-like cerebrobuccal interneuron-2 (CBI-2), which are activated by food (Rosen et al., 1991). These programs are similar to those recorded in behaving animals (Morton and Chiel, 1993a,b; Church and Lloyd, 1994). The first phase of CMPs is the radula protraction phase (Hurwitz et al., 1994, 1996). CBI-2 elicits CMPs by directly activating protraction-phase interneurons and protraction-phase motoneurons (Sanchez and Kirk, 2000; Hurwitz et al., 2003). Protraction-phase motoneurons

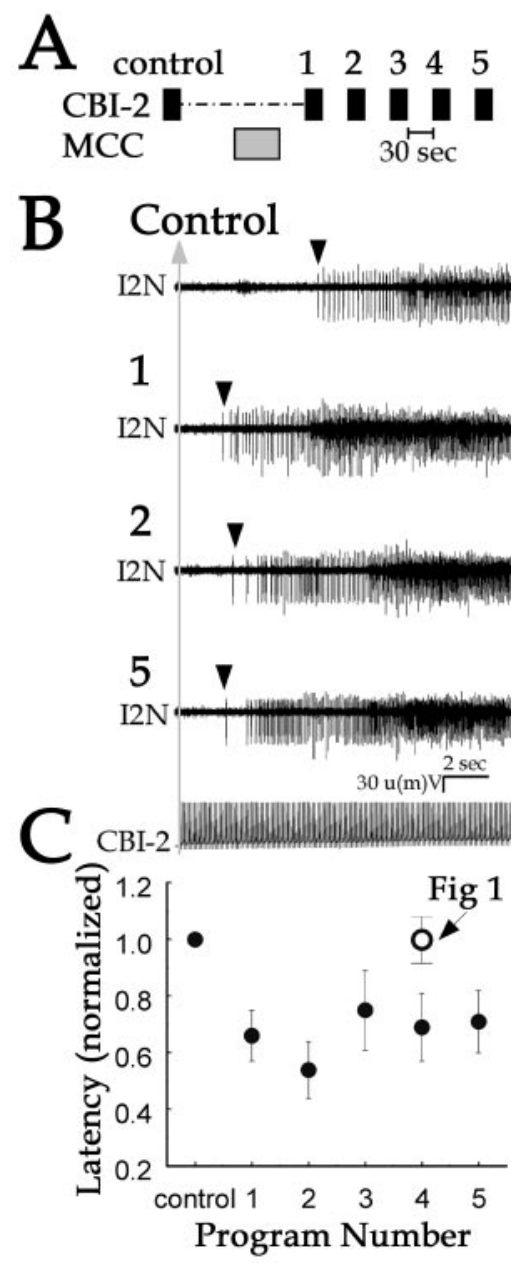

Figure 2. Effect of MCC stimulation when CMPs are elicited frequently. $A$, Paradigm used in the experiment shown in $\mathrm{B}$. $\mathrm{CBI}-2$ was stimulated at $9 \mathrm{~Hz}$ for the duration of the protraction phase to elicit five CMPs with $30 \mathrm{sec}$ intervals. MCC was stimulated at $8 \mathrm{~Hz}$ for 1 min so that the end of the MCC stimulation was 30 sec before the first CBI-2-elicited CMP. At least 20 min of rest were allowed between the control CMP and MCC stimulation. B, All traces are aligned with respect to the beginning of $\mathrm{CBI}-2$ stimulation. Gray arrows indicate the onset of $\mathrm{CBI}-2$ stimulation. Black arrows indicate the onset of activity in the 12 nerve (I2N). 12 recordings are shown for the control CMP. First, second and fifth CMPs elicited subsequent to the end of MCC stimulation. C, Grouped data from five preparations (filled circles). Latency was normalized to the latency of the control CMP. Data are plotted as means \pm SEM $(n=5)$. Data from Figure 1 (the 2 min time point) are plotted for comparison (open circle).

B61/62 receive strong monosynaptic input from CBI-2 and are the first motoneurons recruited into the CMP (Sanchez and Kirk, 2000; Hurwitz et al., 2003). Therefore, we used the time from the beginning of CBI-2 stimulation to the onset of spiking activity of B61/62 as a measure of the latency to the onset of CMPs. Specifically, we monitored B61/62 activity in the I2 nerve, which contains the axons of B61/62 (Hurwitz et al., 1994, 1996). Because shortening of the latency of responses to cued stimuli is the primary indication of the change in the preparatory state, we used the latency to the onset of CMPs as a monitor of the in vitro analog of the preparatory state.

We first investigated whether activity of the MCCs can elicit a change in the in vitro analog of the preparatory state (Fig. $1 A$, paradigm; $B 1-B 3$, representative recordings). I2 nerve activity is shown for the CMP elicited before the onset of MCC stimulation (Fig. 1B1), 1 min after the end of MCC stimulation (Fig. 1B2), and 2 min after the end of MCC stimulation (Fig. 1B3). The time 

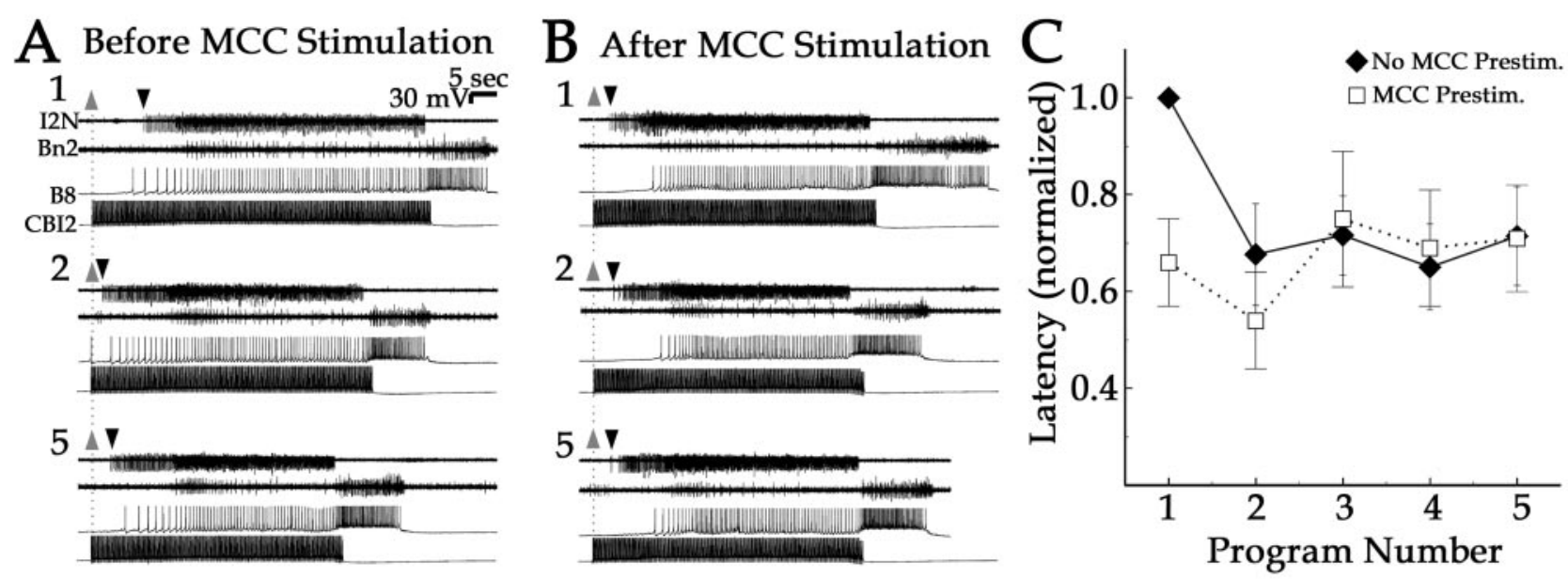

Figure 3. Latency of frequently elicited CMPs with and without MCC prestimulation. $A$, All traces are aligned with respect to CBI-2 stimulation. CBI-2 was used to elicit five CMPs at 30 sec intervals. I2 nerve (I2N) activity, Bn2 activity (retraction monitor), and activity of the radula closer motoneuron B8 are shown for CMPs 1, 2, and 5. Gray arrows indicate the beginning of CBI-2 stimulation. Black arrows indicate the onset of activity in the $\mathrm{I} 2$ nerve. $B$, The MCC was stimulated for $1 \mathrm{~min}$, so that the end of the stimulation was 30 sec before the first stimulation of CBI-2. CBI-2 was used to elicit five CMPs at 30 sec intervals. The order of runs (with and without MCC) was randomized ( 20 min between runs). (BI-2-elicited CMPs 1, 2, and 5 are shown. C, Normalized grouped data from five preparations. The latency of all CMPs after the MCC stimulation was normalized to the first CMP elicited by CBI-2 when CBI-2 stimulation was not preceded by stimulation of the MCC.

between the gray and black arrows represents the latency. Combined results $(n=5)$ (Fig. $1 C)$ show that the latency is shortened 1 min and returns back to control 2 min after MCC stimulation (ANOVA; $F=5.62 ; \mathrm{df}=2.12 ; p<0.05$ ). This effect is consistent with the hypothesized role of the MCCs in the establishment of the preparatory state for consummatory behaviors (Kupfermann et al., 1979).

Although activity of the MCCs drops precipitously at the onset of biting in intact behaving animals (Kupfermann and Weiss, 1982), the latency to biting responses remains short for as long as Aplysia continue biting. Because in the preceding experiment we showed that the effects of the MCCs persist for only $1 \mathrm{~min}$ after the end of MCC stimulation, it is unlikely that activity of the MCCs can explain the long-term maintenance of the decreased biting latency observed in behaving animals. In intact animals (Kupfermann, 1974a), however, bites occur at a much higher frequency than that at which motor programs were elicited in the preceding experiment (once every $4 \mathrm{~min}$ ). This raised the possibility that when elicited frequently, motor programs themselves can maintain the MCC-elicited shortening of the latency. To test this possibility, we prestimulated the MCC and elicited motor programs once every $30 \mathrm{sec}$ rather than once every $4 \mathrm{~min}$. Representative recordings are shown in Figure $2 \mathrm{~B}$. The time between the gray and black arrows represents the latency. Grouped data $(n=5)$ are shown in Figure 2C. In contrast to results in Figure 1, the latency remained short for as long as CBI-2 was stimulated (ANOVA; $F=3.328 ; \mathrm{df}=5.15 ; p<0.05 ; t$ tests with Bonferroni correction between control and the fifth CMP elicited after MCC stimulation, $t=2.791 ; p<0.05)$. The latency to the onset of B61/62 firing in a CMP elicited 2 min after the MCC stimulation (Fig. 1) is plotted in Figure $2 C$ for comparison. Thus, frequent activation of the feeding CPG can maintain the in vitro analog of the preparatory state elicited by stimulation of the MCCs.

In the preceding experiment, frequent stimulation of CMPs resulted in the maintenance of the shortened latency elicited initially by the stimulation of the MCCs. This raised the possibility that even in the absence of MCC stimulation, frequent CBI-2 stimulation is sufficient to produce the persistent shortening of the latency (Fig. 2C). To determine whether repeated stimulation of CBI-2 itself can elicit a change in the in vitro analog of the preparatory state in an MCC-independent manner, we stimulated CBI-2 to elicit five CMPs at 30 sec intervals and analyzed the latency to the onset of $\mathrm{B} 61 / 62$ firing. Representative recordings are shown in Figure $3 \mathrm{~A}$. We prestimulated the MCC to characterize the interaction between MCC-dependent and MCCindependent effects on the latency. Representative recordings are shown in Figure $3 B$. Grouped data $(n=5)$ (Fig. $3 C)$ show that with repeated stimulation of CBI-2, the latency is shortened (ANOVA; $F=3.23$; $\mathrm{df}=4.16 ; p<0.05$ ). The latency remains short for as long as CBI-2 is stimulated repeatedly ( $t$ test with Bonferroni correction for CMP 1 vs CMP 5, $t=2.563$; $p<0.05$ ). When MCC was prestimulated, the latency was shorter than that for CMP 1 elicited by CBI-2 alone (ANOVA; $F=3.328$; $\mathrm{df}=5.15$; $p<0.05 ; t$ test with Bonferroni correction of CMP 1 elicited after MCC to CMP 1 elicited by CBI-2 alone, $t=2.55 ; p<0.05$ ). When MCC was prestimulated, repeated stimulation of CBI-2 did not result in any additional shortening of the latency (ANOVA; $F=$ $0.62 ; \mathrm{df}=4.12 ; p=0.65)$. Furthermore, there were no significant differences between latencies of the last three CMPs regardless of whether or not MCC was prestimulated (ANOVA; $F=0.61$;f $=$ $5.15 ; p=0.68)$. We therefore conclude that although MCCs may first elicit the change in the in vitro analog of the preparatory state, this new state is maintained by MCC-independent mechanisms.

The synapse between CBI-2 and B61/62 exhibits post-tetanic potentiation (PTP) (Sanchez and Kirk, 2000). PTP at this synapse could potentially explain the shortening of the latency when CBI-2 is stimulated frequently (Fig. $3 A$ ). In the following experiment, we investigated whether actions of the MCCs converge on the same synapse. Data from one representative experiment are shown in Figure $4 A 1, A 2$. Figure $4 B$ shows the grouped data $(n=$ $5)$. The PSPs are potentiated after the end of the MCC stimulation, remain potentiated for at least $1 \mathrm{~min}$, and recover within 2 min. Note that the time course of this effect parallels closely that of the effect of the MCCs on the latency (Fig. 1).

It has been suggested that PTP at the CBI-2 to B61/62 synapse is mediated presynaptically (Sanchez and Kirk, 2000). To characterize the site of action of the MCCs at the CBI-2 to B61/62 

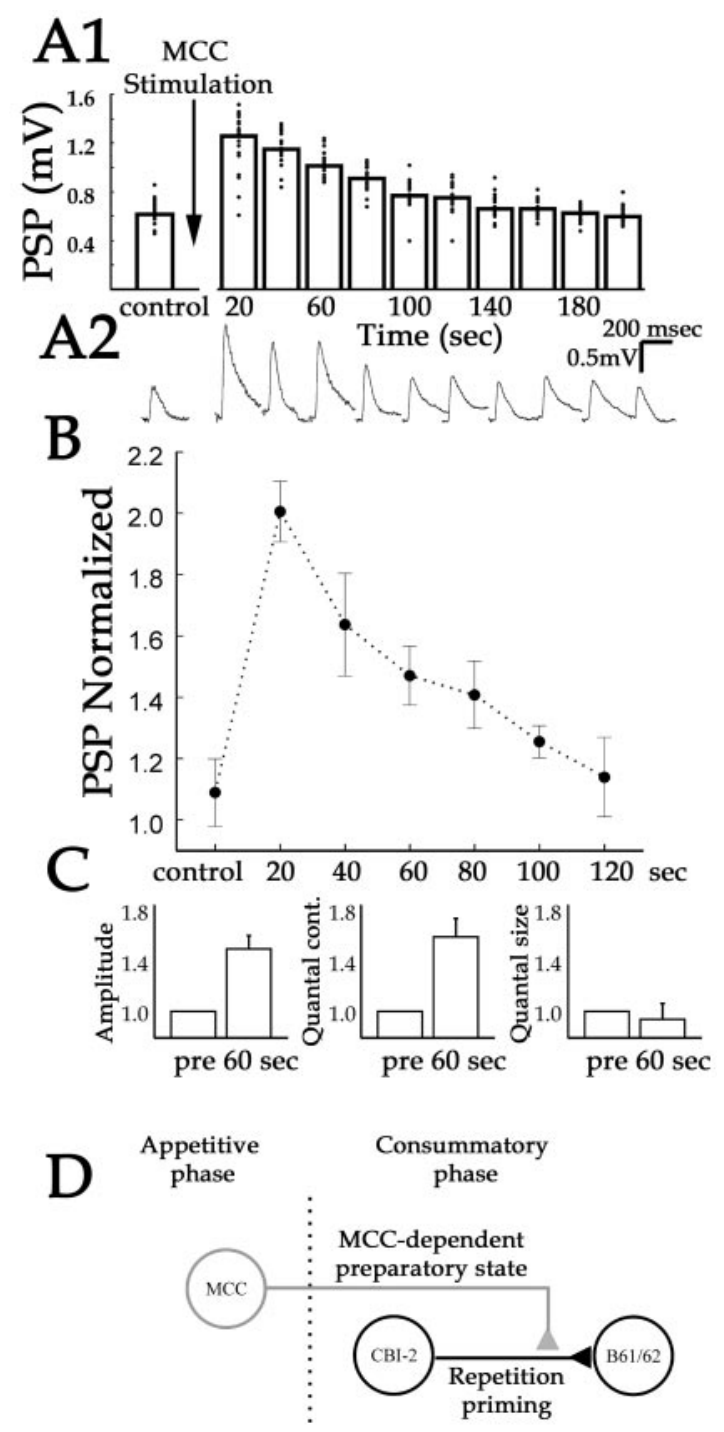

Figure 4. MCC-elicited potentiation of CBI-2 to B61/62 PSPs. CBI-2 was stimulated to fire action potentials at $2.5 \mathrm{~Hz}$, and PSPs were recorded in the soma of ipsilateral $\mathrm{B} 61 / 62$. MCC was then stimulated at $8 \mathrm{~Hz}$ for $1 \mathrm{~min}$. A1, PSPs elicited by CBI-2 subsequent to the end of MCC stimulation were binned into $20 \mathrm{sec}$ bins. Dots represent individual measurements, and bars represent the average for each bin. $A 2$, Examples of individual representative PSPs recorded in the same experiment as $A 1$. $B$, Normalized grouped data from five preparations. Data were binned into 20 sec bins as in $A$. Data are plotted as the average \pm SEM $(n=5)$ for each bin. $C$, Results of the variance method analysis of the data in $B$. Quantal content and quantal number were estimated for the control measurements and the measurements in the $40-60$ bin. All measurements were normalized to those estimated in the control group. Data are plotted as means $\pm \operatorname{SEM}(n=5)$. D, Diagrammatic representation of connections and actions of MCC, CBI-2, and B61/62. The dotted line represents the transition between the appetitive phase and the consummatory phase. $\mathrm{MCC}$ is active primarily during the appetitive phase and facilitates the CBI-2 to B61/62 synapse that becomes activated in the consummatory phase that follows (MCCdependent preparatory state). As the consummatory responses are generated repetitively, the MCC-dependent facilitation of the CBI-2 to B61/62 synapse is replaced by the homosynaptic PTP at the same synapse (repetition priming). The black triangle indicates a fast synaptic connection; the gray triangle indicates a modulatory connection.

synapse, we applied the variance method (see Materials and Methods) to control PSPs as well as to PSPs elicited in the 40-60 sec bin after the end of MCC stimulation because of the results shown in Figure 1. The results in Figure $4 C$ show that in the $40-60 \mathrm{sec}$ bin, the calculated number of quanta of neurotransmitter (quantal content) is increased ( $t=5.26$; $p<0.05$ ), whereas the calculated efficacy of neurotransmitter (quantal size) stays the same $(t=0.48 ; p=0.66)$. We assume that this estimated increase in the number of quanta of transmitter indicates that the MCCs act presynaptically to facilitate the CBI-2 to B61/62 synapse. Thus, homosynaptic plasticity stemming from activation of the command-like CBI-2 neuron and heterosynaptic plasticity stemming from activation of the MCCs appears to converge on the presynaptic terminal of CBI-2 to mediate the shortening of the latency to the onset of CMPs.

\section{Discussion}

In this study, we used the latency to the onset of CMPs as a monitor of the in vitro analog of the preparatory state and characterized the neuronal mechanisms that underlie the changes in this state.

Neuronal mechanisms of in vitro analog of preparatory states Several lines of indirect evidence implicated activity of the MCCs in the establishment of the preparatory state both for biting behavior in intact animals (Weiss and Kupfermann, 1976; Kupfermann and Weiss, 1982; Rosen et al., 1989) and for the neuronal analog of biting in the isolated CNS (Morgan et al., 2000). In this study, we extend these previous suggestions by demonstrating that MCC stimulation exerts persistent effects on the feeding circuitry. This is of crucial importance because in intact feeding animals, the MCCs are active primarily before the onset of biting, and therefore to influence biting responses, the effects of the MCCs must outlast their activity. On the basis of MCC lesion studies, it has been hypothesized that as the meal progresses, the initial preparatory state elicited by the MCCs may be maintained by MCC-independent mechanisms (Rosen et al., 1989). In this study, we demonstrate that both MCC-dependent and MCCindependent mechanisms participate in the establishment and maintenance of the in vitro analog of the preparatory state, respectively (Fig. 3).

We used the in vitro analog of the preparatory state to characterize synaptic mechanisms that may underlie its establishment and maintenance. We focused on the CBI-2 to B61/62 synapse because of its crucial role in controlling the latency to the onset of CBI-2-elicited CMPs. Consistent with its effect on the latency to the onset of CMPs (Fig. 1), we found that the MCCs facilitate the CBI-2 to B61/62 synapse (Fig. 4). The time course of this facilitation parallels the effect of the MCCs on the latency to the onset of CMPs (Figs. 1, 4). However, actions of the MCCs do not account for the long-term maintenance of the preparatory state that is observed when motor programs are elicited frequently. This MCC-independent long-term maintenance may be mediated by PTP (Sanchez and Kirk, 2000) at the CBI-2 to B61/62 synapse. Thus, both MCC-dependent and MCC-independent mechanisms converge on the same synapse to mediate changes in the in vitro analog of the preparatory state. Notably, both PTP at the CBI-2 to B61/62 synapse (Sanchez and Kirk, 2000) and heterosynaptic facilitation of the same synapse by the MCCs appear to operate through presynaptic mechanisms.

\section{Beyond Aplysia}

Preparatory states are a marked feature of behavior of different species, suggesting that similar neuronal mechanisms may be involved. Several studies have implicated locus ceruleus neurons (LCNs) in the preparatory states of vertebrates. LCNs and MCCs are similar in several respects. Both LCNs (Foote et al., 1980) and MCCs (Kupfermann and Weiss, 1982) are activated preferentially by stimuli that elicit orienting/preparatory responses. Activity of both LCNs (Foote et al., 1980) and MCCs (Kupfermann 
and Weiss, 1982) correlates with the arousal state of the animal. Both LCNs and MCCs exert widespread modulatory actions.

Several lines of evidence suggest that LCNs are involved in mediating changes in the preparatory states in primates. In visual discrimination tasks, LCNs are activated phasically by salient stimuli before the onset of action (Aston-Jones et al., 1994; Usher et al., 1999). Different parameters of the activity of LCNs during this task correspond to different levels of an animal's performance and response latency (Aston-Jones et al., 1994; Usher et al., 1999). Pharmacological manipulations of LCNs are consistent with their involvement in mediating preparatory states (Aston-Jones et al., 1999). Through the use of computational models, it has been suggested that LCNs mediate preparatory states by increasing the gain in the behavior-generating networks (Usher et al., 1999; Usher and Davelaar, 2002). Our results are in direct agreement with the predictions of this theoretical model, because we have demonstrated experimentally that the MCCs facilitate synaptic transmission between CBI-2, which initiates behavior, and its postsynaptic partner, which implements it. LCNs may not be the sole mediators of preparatory states during visual discrimination tasks, because activity of the LCNs decreases with repeated presentation of the same stimulus, whereas performance improves (Aston-Jones et al., 1994). Perhaps in primates, through repetition priming, the behavior-generating networks can maintain the preparatory state in the absence of the activity of LCNs, as the feeding network does in Aplysia in the absence of MCC activity.

\section{Concluding remarks}

We found that both MCC-dependent and MCC-independent mechanisms of latency shortening converge on the presynaptic site of the CBI-2 to B61/62 synapse. In principle, a number of distinct mechanisms could be used to produce latency shortening. For instance, one obvious alternative would be to increase the excitability of B61/62. However, because the feeding network of Aplysia is multitasking, such a mechanism could also affect the response of B61/62 to inputs that elicit other behaviors. One important feature of the preparatory states, however, is that they facilitate specific responses selectively. Convergence of the MCCdependent and MCC-independent mechanisms on the same presynaptic site may act to ensure this specificity of preparatory states on the neuronal level.

\section{References}

Aston-Jones G, Rajkowski J, Kubiak P, Alexinsky T (1994) Locus ceruleus neurons in monkey are selectively activated by attended cues in a vigilance task. J Neurosci 14:4467-4480.

Aston-Jones G, Rajkowski J, Cohen J (1999) Role of locus coeruleus in attention and behavioral flexibility. Biol Psychiatry 46:1309-1320.

Church PJ, Lloyd PE (1994) Activity of multiple identified motor neurons recorded intracellularly during evoked feedinglike motor programs in Aplysia. J Neurophysiol 72:1794-1809.

Connolly JD, Goodale MA, Menon RS, Munoz DP (2002) Human fMRI evidence for the neural correlates of preparatory set. Nat Neurosci 5:1345-1352.

Foote SL, Aston-Jones G, Bloom FE (1980) Impulse activity of locus coeruleus neurons in awake rats and monkeys is a function of sensory stimulation and arousal. Proc Natl Acad Sci USA 77:3033-3037.

Hubbard L, Quastel DM, Llinas R (1969) Electrophysiological analysis of synaptic transmission (Hubbard JI, Llinas RR, Quastel DMJ, eds). Baltimore: Williams \& Wilkins.

Hurwitz I, Goldstein RS, Susswein AJ (1994) Compartmentalization of pattern-initiation and motor functions in the $\mathrm{B} 31$ and $\mathrm{B} 32$ neurons of the buccal ganglia of Aplysia californica. J Neurophysiol 71:1514-1527.

Hurwitz I, Neustadter D, Morton DW, Chiel HJ, Susswein AJ (1996) Activity patterns of the B31/B32 pattern initiators innervating the I2 muscle of the buccal mass during normal feeding movements in Aplysia californica. J Neurophysiol 75:1309-1326.

Hurwitz I, Kupfermann I, Weiss KR (2003) Fast synaptic connections from cerebro-buccal interneurons (CBIs) to pattern generating interneurons in Aplysia: initiation and modification of buccal motor programs. J Neurophysiol 89:2120-2136.

Kupfermann I (1974a) Feeding behavior in Aplysia: a simple system for the study of motivation. Behav Biol 10:1-26.

Kupfermann I (1974b) Dissociation of the appetitive and consummatory phases of feeding behavior in Aplysia: a lesion study. Behav Biol 10:89-97.

Kupfermann I, Weiss KR (1982) Activity of an identified serotonergic neuron in free moving Aplysia correlates with behavioral arousal. Brain Res 241:334-337.

Kupfermann I, Cohen JL, Mandelbaum DE, Schonberg M, Susswein AJ, Weiss KR (1979) Functional role of serotonergic neuromodulation in Aplysia. Fed Proc 38:2095-2102.

Morgan PT, Perrins R, Lloyd PE, Weiss KR (2000) Intrinsic and extrinsic modulation of a single central pattern generating circuit. J Neurophysiol 84:1186-1193

Morton DW, Chiel HJ (1993a) In vivo buccal nerve activity that distinguishes ingestion from rejection can be used to predict behavioral transitions in Aplysia. J Comp Physiol [A] 172:17-32.

Morton DW, Chiel HJ (1993b) The timing of activity in motor neurons that produce radula movements distinguishes ingestion from rejection in Aplysia. J Comp Physiol [A] 173:519-536.

Rosen SC, Weiss KR, Goldstein RS, Kupfermann I (1989) The role of a modulatory neuron in feeding and satiation in Aplysia: effects of lesioning of the serotonergic metacerebral cells. J Neurosci 9:1562-1578.

Rosen SC, Teyke T, Miller MW, Weiss KR, Kupfermann I (1991) Identification and characterization of cerebral-to-buccal interneurons implicated in the control of motor programs associated with feeding in Aplysia. J Neurosci 11:3630-3655.

Sanchez JA, Kirk MD (2000) Short-term synaptic enhancement modulates ingestion motor programs of Aplysia. J Neurosci 20:RC85(1-7).

Schacter DL, Buckner RL (1998) Priming and the brain. Neuron 20:185-195.

Susswein AJ, Weiss KR, Kupfermann I (1984) Internal stimuli enhance feeding behavior in the mollusc Aplysia. Behav Neural Biol 41:90-95.

Usher M, Davelaar EJ (2002) Neuromodulation of decision and response selection. Neural Netw 15:635-645.

Usher M, Cohen JD, Servan-Schreiber D, Rajkowski J, Aston-Jones G (1999) The role of locus coeruleus in the regulation of cognitive performance. Science 283:549-554.

Weiss KR, Kupfermann I (1976) Homology of the giant serotonergic neurons (metacerebral cells) in Aplysia and pulmonate molluscs. Brain Res 117:33-49.

Wiggs CL, Martin A (1998) Properties and mechanisms of perceptual priming. Curr Opin Neurobiol 8:227-233. 